\title{
Role of diffusion-weighted MRI in evaluation of pediatric musculoskeletal soft tissue masses
}

\author{
Mohamed Ali El-Adalany' ${ }^{1}$ Nevertiti Kamal El-Din Eid ${ }^{1}$, Salma Othman ${ }^{2}$ and Dina El-Metwally ${ }^{1 *}$
}

\begin{abstract}
Background: In pediatric patients, soft tissue masses encompass a wide heterogeneous group of benign and malignant lesions. MRI is a powerful diagnostic tool in the workup of soft tissue tumors in children, and it helps in characterization of lesion and evaluation of the extent of the lesion. However, conventional MRI techniques are not specific in differentiating benign from malignant lesions. So to improve characterization of tumors, DWI was added to MRI techniques as it increases sensitivity and specificity by detecting the micro-diffusion changes of water into intra- and extracellular spaces. The aim of this work was to highlight the diagnostic value of DWI in detection and characterization of different musculoskeletal soft tissue masses in pediatrics.

Results: There was a statistically significant difference regarding the mean ADC value of benign and malignant masses $\left(P\right.$ value $\left.=0.001^{*}\right)$. The mean ADC value for all benign masses $(n=41)$ was $1.495 \pm 0.55 \mathrm{SD} \times 10^{-3} \mathrm{~mm}^{2} / \mathrm{s}$, while the mean ADC value for all malignant masses $(n=21)$ was $0.449 \pm 0.27 \mathrm{SD} \times 10^{-3} \mathrm{~mm}^{2} / \mathrm{s}$. The cutoff ADC value between benign and malignant masses was $0.88 \times 10^{-3} \mathrm{~mm}^{2} / \mathrm{s}$. This cutoff ADC value has sensitivity of $100.0 \%$, specificity of $92.3 \%$, PPV of $66.7 \%$, NPV of $100.0 \%$ and diagnostic accuracy of $93.3 \%$.

Conclusion: In pediatric patients, DWI is an innovative valuable noninvasive imaging technique for characterization of musculoskeletal soft tissue masses and discrimination between benign and malignant masses.
\end{abstract}

Keywords: MRI, DWI, Soft tissue, Masses, Benign, Malignant, Pediatrics

\section{Background}

Soft tissue masses and osseous lesions are commonly encountered in pediatric patients. Musculoskeletal soft tissue masses encompass a wide heterogeneous group of benign and malignant lesions that developed from connective tissue other than bone. Although benign in most cases, a significant proportion of pediatric musculoskeletal soft tissue masses constitutes malignant tumors [1]. Patients who have a soft tissue tumor or tumor-like mass usually presented with non-specific clinical findings such as soft tissue swelling or pain. In many cases, a detailed history and physical examination are sufficient to make a diagnosis, but in circumstances when the diagnosis is

*Correspondence: elmetwallydina@gmail.com

${ }^{1}$ Faculty of Medicine, Mansoura University, Mansoura, Egypt Full list of author information is available at the end of the article uncertain, imaging can be very helpful to characterize the mass [2].

MRI is a powerful diagnostic tool in the workup of soft tissue tumors in children due to its high spatial and contrast resolution [3]. However, there is often an overlap in the MRI appearance of benign and malignant soft tissue tumors, resulting in decreasing the diagnostic confidence. The specificity of conventional MRI in differentiating benign from malignant soft tissue tumors was variable among previous studies, presumably because benign and malignant soft tissue tumors may share the same morphologic features regarding the size, margin, location, signal intensities and contrast enhancement $[4$, 5].

So to improve characterization of tumors, DWI was added to MRI techniques as it increases sensitivity and specificity by detecting the micro-diffusion changes of water into intra- and extracellular spaces [4]. DWI is a 
non-enhanced functional MRI technique that reflects differences in the Brownian motion of water caused by variations in tissue microstructure. The apparent diffusion coefficient (ADC) is a quantitative measure of Brownian motion: Low ADC values reflect highly cellular microenvironment in which diffusion is limited by an abundance of cell membranes, whereas high ADC values are observed in cellular regions that allow free diffusion of water molecules. Thus, DWI offers quantitative functional assessment of cellularity at the molecular level, with the potential to help differentiate between benign and malignant lesions $[6,7]$.

DWI was considered an innovative imaging technique for characterization of musculoskeletal lesions which is particularly suitable for pediatric patients and is expected to aid in decision making to perform or postpone biopsy with higher diagnostic confidence. Also, it has particular value to avoid invasive diagnostic procedures in benign pathologies [8]. Several previous studies [6, 9-11] evaluated the role of DWI in assessment of soft tissue tumors; to the best of our knowledge, this study is the first to examine musculoskeletal soft tissue masses (benign masses as hemangioma, lymphangioma and Baker's cyst and malignant masses as rhabdomyosarcoma, lymphoma and Ewing sarcoma) in pediatric population using DWI specifically.

\section{Aim of the work}

The purpose of this study was to highlight the diagnostic value of diffusion-weighted imaging (DWI) in detection, delineation and characterization of different musculoskeletal soft tissue masses in pediatric population.

\section{Methods}

\section{Patient's demographic data}

This prospective study was approved by our institutional ethics committee, and subjects agreed to participate and publicate images in this study with a written consent obtained from their guardians. This study was performed during the period from December 2019 till June 2021. It included 62 pediatric patients, 37 females (59.7\%) and 25 males $(40.3 \%)$, and their age ranged from 2 to 16 years (mean $\pm \mathrm{SD}=5.39 \pm 5.31 \mathrm{SD}$ ). According to the results of histopathological examinations, we had 41 benign lesions and 21 malignant lesions.

\section{Inclusion criteria}

Inclusion criteria are as follows: pediatric patients clinically suspected to have musculoskeletal soft tissue masses.

\section{Exclusion criteria}

Exclusion criteria are as follows: uncooperative patients or those who had contraindications to magnetic resonance imaging as patients with cochlear implants or prosthetic valve, patients who received chemotherapy or radiotherapy prior to the MRI study and patients who underwent total excision of the tumor or excisional biopsy prior to the MRI study.

\section{MRI technique}

MRI examinations were performed for all patients using a 1.5-T MRI unit (Acheiva; Philips Medical Systems, Best, Netherlands). Throughout the examination, most of cases were in supine position using a body phased-array coil, a standard head and neck coil, flexed coil or different surface coils according to patient's size and examined portion. Before DWI, all patients had routine pulse sequences for conventional non-contrast T1-weighted fast spin-echo images, T2-WI, STIR or fat sat MRI images which were obtained in different planes.

- The predetermined examination protocol was applied to all patients that included the following:

(1) $\mathrm{T}_{1} \mathrm{WI}(\mathrm{TR} / \mathrm{TE}=400-700 / 14-30$, FOV 20-35) and $\mathrm{T}_{2} \mathrm{WI}(\mathrm{TR} / \mathrm{TE}=2800-4500 / 80-120$, FOV 20-35) in axial, coronal and /or sagittal planes, slice thickness ranging from 4 to $10 \mathrm{~mm}$, interslice gap of $2-3 \mathrm{~mm}$, the matrix for all sequences till $512 * 512$.

(2) At least one fat-saturated sequence or short inversion recovery (STIR) sequence (TR/ $\mathrm{TE}=4000-5600 / 18-40$, FOV 20-35).

- The mean time of conventional MR examination was approximately 15-20 min.

- DWI was obtained in the axial plane by using a nonbreath-hold single-shot spin-echo EPI sequence using different image parameters considering (repetition time, echo time, number of signals acquired, percent phase, field of view, slice thickness, gap, flip angle, echo-train length and matrix) according to site and size of each mass, e.g., shoulder mass parameters $\left(\mathrm{TR} / \mathrm{TE}=692 / 69\right.$, flip angle $90^{\circ}$, slice thickness $6 \mathrm{~mm}$, gap $0.6 \mathrm{~mm}$., FOV 22, EPI factor 25). The b-values used in this study were 0,400 and $800 \mathrm{~s} / \mathrm{mm}^{2}$. The mean time of the DW examination for each patient was approximately 3-5 min.

- ADC maps were automatically generated by the MRI software on the basis of the images obtained. 
On ADC maps, regions of interest (ROI) were manually positioned and placed over the most homogenous portion of solid and cystic parts of the musculoskeletal soft tissue masses to determine the ADC value, and then, all values were automatically calculated and expressed by $\times 10^{-3} \mathrm{~mm}^{2} / \mathrm{s}$.

- The representative value used in data and statistical analysis was the mean value.

1. Image analysis DWI analysis included qualitative and quantitative analysis.

\section{A. Qualitative analysis}

It refers to the visual assessment of the signal intensity of the masses. The masses were classified into masses with low signal and masses with high signal in comparison with the signal intensity of adjacent skeletal muscles. The mass signal on DWI was correlated with the corresponding signal on the automatically generated ADC maps.

Masses with free diffusion showed low signal on DWI and high signal on ADC map, while restricted diffusion was seen as high signal on high b value (800) DWI and low signal on corresponding ADC map. Masses with T2 shine through effect showed high signal in both DWIs and ADC maps.

\section{B. Quantitative analysis}

ADC values were calculated from the ADC maps. For calculating the mean ADC value of the solid part of a mass, a ROI was drawn on the ADC map and placed on the solid area of the mass, apart from any necrotic areas in mixed masses. Concerning homogenous appearing masses, a circular ROI (1-2) $\mathrm{cm}$ was placed on the center of the mass mostly or measuring three $\mathrm{ADC}$ values then the mean $\mathrm{ADC}$ value was calculated.

\section{Standard of reference:}

Our MRI results were compared with histopathological results after biopsy (Tru-cut biopsy from solid masses and fine needle aspiration cytology (FNAC) from cystic masses), and then, diagnostic specificity and sensitivity were estimated by statistical analysis.

3. Statistical analysis and data interpretation
Data were fed to the computer and analyzed using IBM SPSS Corp., Released 2013, IBM SPSS Statistics for Windows, version 22.0, Armonk, NY: IBM Corp. Qualitative data were described using number and percent. Quantitative data were described using median (minimum and maximum) for nonparametric data and mean and standard deviation for parametric data after testing normality using Shapiro-Wilk test. Significance of the obtained results was judged at the 0.05 level. The diagnostic performance of a test or the accuracy of a test to discriminate diseased cases from non-diseased cases was evaluated using receiver operating characteristic (ROC) curve analysis. Sensitivity and specificity were detected from the curve, and PPV, NPV and accuracy were calculated through cross-tabulation.

\section{Results}

This study included 62 pediatric patients, 37 females (59.7\%) and 25 males (40.3\%), and their age ranged from 2 to 16 years (mean $\pm \mathrm{SD}=5.39 \pm 5.31 \mathrm{SD}$ ). According to the results of histopathological examinations, we had 41 benign lesions and 21 malignant lesions, and out of the 41 benign lesions, we had 9 cases with lipoma, 8 cases with congenital hemangioma (Fig. 1), 5 cases with lymphangioma (Fig. 2), 4 cases with Baker's cyst, 4 cases with fibroma, 2 cases with vascular malformation, 6 cases with abscess, 2 cases with pigmented villonodular synovitis (Fig. 3) and 1 case with lipoblastoma. Out of the 21 malignant lesions, we had 8 cases with rhabdomyosarcoma, 7 cases with Ewing sarcoma and 6 cases with lymphoma.

In this study, 53.3\% of cases show free diffusion in DWI with high signal in ADC map, while $46.7 \%$ of cases show restricted diffusion in DWI with low signal recorded in ADC map as shown in Table 1.

Regarding the measured ADC values, there was a statistically significant difference between benign and malignant masses $(P$ value $=0.001 *)$. Out of the 62 cases, the mean ADC value for all benign masses $(n=41)$ was $1.495 \pm 0.55 \mathrm{SD} \times 10^{-3} \mathrm{~mm}^{2} / \mathrm{s}$, which was significantly higher than the mean ADC value for all malignant masses $(n=21)$ that was $0.449 \pm 0.27 \mathrm{SD} \times 10^{-3} \mathrm{~mm}^{2} / \mathrm{s}$ as shown in Table 2.

According to ADC values distribution, the highest recorded mean ADC was that of Baker's cyst $(2.6440 \pm 0.10889 \mathrm{SD}) \times 10^{-3} \mathrm{~mm}^{2} / \mathrm{s}$ followed by that of lymphangioma $(2.2100 \pm 0.03960 \mathrm{SD}) \times 10^{-3} \mathrm{~mm}^{2} / \mathrm{s}$, while the least recorded mean ADC value was that of the malignant cases in the study which were rhabdomyosarcoma $(0.4498 \pm 0.27050 \mathrm{SD}) \times 10^{-3} \mathrm{~mm}^{2} / \mathrm{s}$ with a statistically significant difference $\left(P<0.001^{*}\right)$ as shown in Fig. 4 and Table 3. 


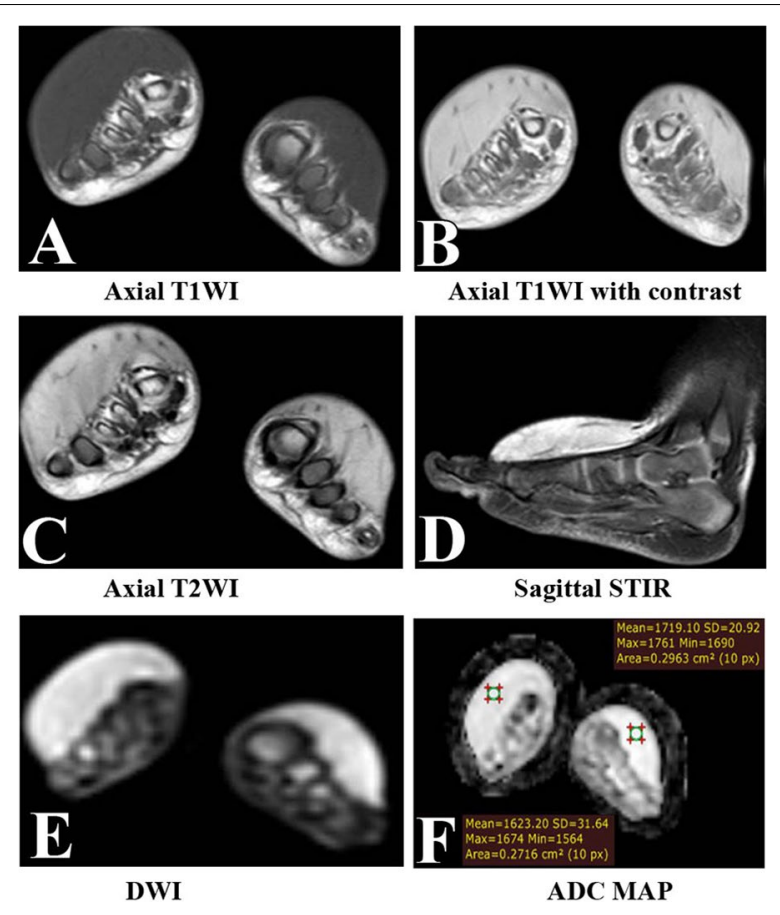

Fig. 1 A 1-year-old male patient, presented with bilateral dorsal foot swellings. A, B Axial T1WI and axial T1WI with contrast: Fairly defined near symmetrical diffuse subcutaneous soft tissue thickening low signal intensity is seen on the dorsal aspect of both feet with no definite cortical bone disruption or significant abnormal marrow signal. It measures about $2 \mathrm{~cm}$ on the right side and $11 \mathrm{~mm}$ on the left side in its maximum dimension with intense enhancement in post-contrast series. C, D Axial T2WI and sagittal STIR right foot: Both swellings show high signal intensity in T2WI, not suppressed on STIR with linear signal void patterns (blood threads). E Axial DWl: Each swelling elicits high signal intensity. F Axial DW-ADC map image: high signal intensity recorded for each swelling with high mean ADC value $\left(\right.$ right $\left.=1.623 \times 10^{-3} \mathrm{~mm}^{2} / \mathrm{s}\right)$ and $\left(\right.$ left $\left.=1.719 \times 10^{-3} \mathrm{~mm}^{2} / \mathrm{s}\right)$. Pathological result was bilateral dorsal foot hemangiomas

In this study, ROC curve analysis of the mean ADC in benign versus malignant masses yielded a confidence interval (CI) of $95 \%$ and area under curve (AUC) of $0.981 \times 10^{-3} \mathrm{~mm}^{2} / \mathrm{s}$. The cutoff point for all mean ADC value was $0.88 \times 10^{-3} \mathrm{~mm}^{2} / \mathrm{s}$ with a high statistically significant difference between benign and malignant masses $\left(P \leq 0.001^{*}\right)$, sensitivity of $100.0 \%$, specificity of $92.3 \%$, PPV of $66.7 \%$, NPV of $100.0 \%$ and overall diagnostic accuracy of $93.3 \%$ as shown in Fig. 5 and Table 4.

\section{Discussion}

A wide spectrum of entities may give rise to musculoskeletal soft tissue masses in pediatrics, including neoplastic and non-neoplastic masses. MRI plays a significant role in characterization of soft tissue tumors, yet conventional techniques lack specificity for proper differentiation between benign and malignant masses [12]. DWI is a noninvasive method for investigation of tumor histology

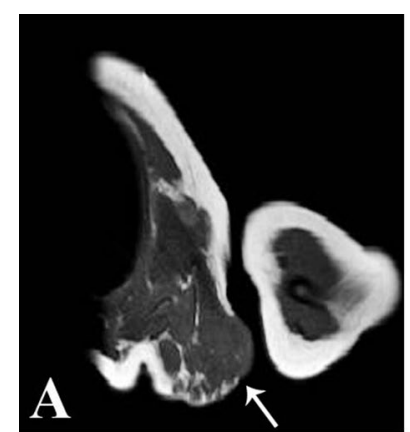

Axial T1WI

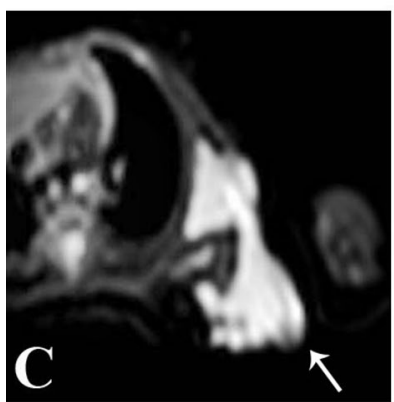

DWI

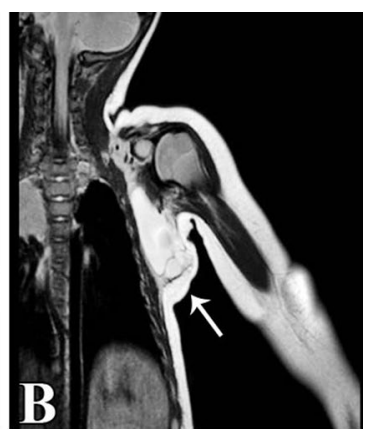

Coronal T2WI

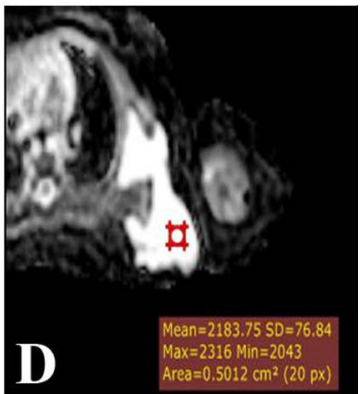

ADC MAP
Fig. 2 A 6-month-old male patient, presented with a painless mass at the left axilla. A Axial T1WI: A large lobulated multilocular cystic soft tissue mass elicits low signal intensity seen in the left axillary region with no intrathoracic extension. B Coronal T2Wl: The mass elicits high signal intensity. C Axial DWI with $b$ value of $800 \mathrm{~s} / \mathrm{mm}^{2}$ : The mass appears of low signal intensity. D Axial ADC map image: bright signal intensity recorded with high mean $\mathrm{ADC}$ value $\left(2.183 \times 10^{-3} \mathrm{~mm}^{2} / \mathrm{s}\right)$. Pathological result was a case of lymphangioma

and used effectively for differentiation between benign and malignant masses [13].

In this study, there was a statistically significant difference between the mean ADC value of benign and malignant masses $\left(P=0.001^{*}\right)$, and the mean ADC value for benign masses was $1.495 \pm 0.55 \mathrm{SD} \times 10^{-3} \mathrm{~mm}^{2} / \mathrm{s}$ versus $(0.449 \pm 0.27 \mathrm{SD}) \times 10^{-3} \mathrm{~mm}^{2} / \mathrm{s}$ for the malignant masses. This is in agreement with previous studies, and one study stated that the mean ADC value of benign masses was $2.31 \pm 1.29 \times 10^{-3} \mathrm{~mm}^{2} / \mathrm{s}$ versus $0.90 \pm 0.70 \times 10^{-3} \mathrm{~mm}^{2} / \mathrm{s}$ for malignant masses $(P<0.001)$ [13]. Other studies reported that there was a statistically significant difference between the mean ADC value of benign and malignant soft tissue tumors $(P<0.05)[5,14]$.

The reported significant difference in our study also matched with that in the study of Zou et al. [15] that showed a significant decrease in the mean ADC value of patients with malignant soft tissue tumors in comparison with the ADC values obtained in patients with benign soft tissue tumors $(P<0.001)$. Their reported mean ADC value of benign soft tissue tumors was 


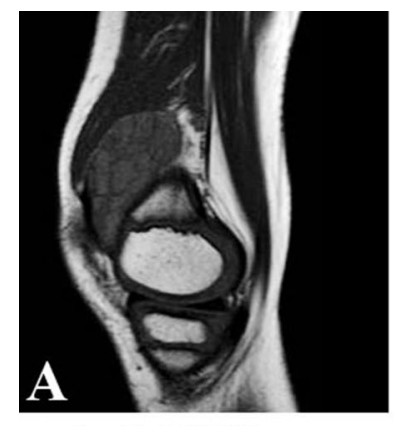

Sagittal T1WI

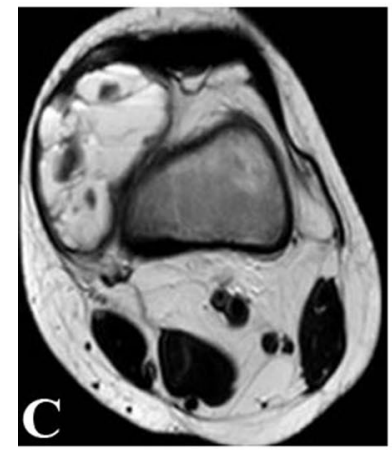

Axial T2WI

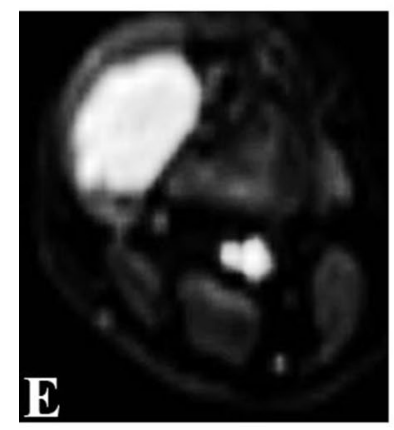

DWI

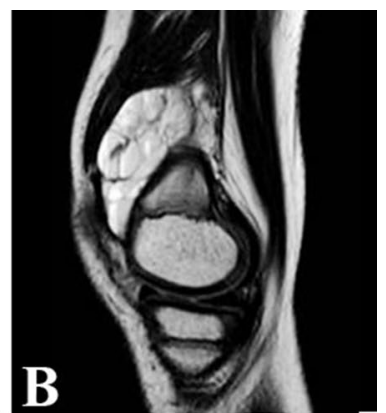

Sagittal T2WI

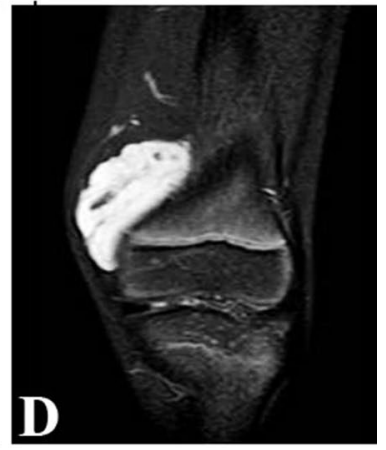

Coronal STIR

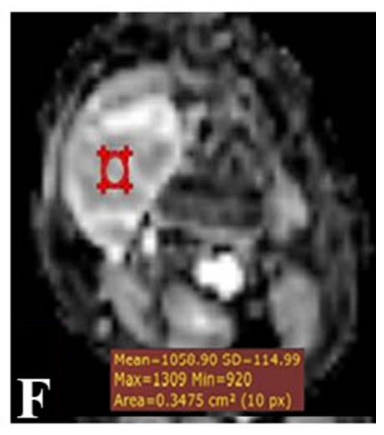

ADC MAP
Fig. 3 A 7-year-old female patient, presented with a painless mass at the anteromedial aspect of the left knee. A Sagittal T1WI: A localized well-defined multilocular cystic mass of low signal intensity is seen in the anteromedial aspect of the left knee with intact articular cartilages, peri-articular musculature, ligaments and preserved intervening fat planes. B, C Sagittal and axial T2Wl: the mass elicits intermediate to high signal intensity. D Coronal STIR: the mass appears of high signal intensity with signal void structures seen within. E Axial DWI: The mass elicits high signal intensity. $\mathbf{F}$ Axial DW-ADC map image: low signal intensity recorded with mean ADC value $\left(1.058 \times 10^{-3} \mathrm{~mm}^{2} / \mathrm{s}\right)$. Pathological result was localized extra-articular pigmented villonodular synovitis

$1.73 \times 10^{-3} \mathrm{~mm}^{2} / \mathrm{s}$, while the mean $\mathrm{ADC}$ value of malignant soft tissue tumors was $0.8 \times 10^{-3} \mathrm{~mm}^{2} / \mathrm{s}$. Also our results matched with the previous study where the reported mean $\mathrm{ADC}$ value for malignant soft tissue tumors was $0.90 \pm 0.32 \times 10^{-3} \mathrm{~mm}^{2} / \mathrm{s}$ with a statistically significant difference between benign and
Table 1 ADC and DWI expression among the studied cases

\begin{tabular}{lll}
\hline & $\mathbf{N}=\mathbf{3 0}$ & $\%$ \\
\hline ADC & & \\
Low signal & 29 & 46.7 \\
High signal & 33 & 53.3 \\
ADC $\left(\times 10^{-3} \mathrm{~mm}^{2} / \mathrm{s}\right)$ & & \\
Mean \pm SD & $1.36 \pm 0.63$ & \\
Median (range) & $(0.2-2.72)$ & \\
DWl & & 53.3 \\
Free & 33 & 46.7 \\
Restricted & 29 & \\
\hline
\end{tabular}

malignant masses $(P<0.001)$ [16]. The same significant difference between benign and malignant soft tissue tumors was also recorded in the previous study $(P<0.0001)$ [17]. Other study showed that malignant rhabdomyosarcoma had a characteristically low ADC value of $0.71 \pm 0.15 \times 10^{-3} \mathrm{~mm}^{2} / \mathrm{s}$ with a statistically significant difference between benign and malignant masses $(P<0.05)[9]$. Another study stated that there was a statistically significant difference between the mean ADC value of benign and malignant masses $(P<0.001)$ (mean $\pm \mathrm{SD}, 1.43 \pm 0.56 \times 10^{-3} \mathrm{~mm}^{2} / \mathrm{s}$ for benign masses versus $0.74 \pm 0.18 \times 10^{-3} \mathrm{~mm}^{2} / \mathrm{s}$ for malignant masses) [18].

Among benign masses, as they are composed primarily of free water, we found that the highest mean ADC value recorded was that of Baker's cyst cases (2.6440 \pm 0.109 SD) $\times 10^{-3} \mathrm{~mm}^{2} / \mathrm{s}$. This is in agreement with results of other study [13] in which the mean ADC value of benign cystic masses was the highest among benign cases $(2.61 \pm 0.35) \times 10^{-3} \mathrm{~mm}^{2} / \mathrm{s}$. Also our results are in agreement with Khedr et al's [17] where the highest ADC value among benign cases was recorded in cases of ganglion cyst $(2.8 \pm 0.23) \times 10^{-3} \mathrm{~mm}^{2} / \mathrm{s}$ and cystic neurofibroma $(2.5 \pm 0.04) \times 10^{-3} \mathrm{~mm}^{2} / \mathrm{s}$. It was reported that in cases in which a cyst is suspected, ADC mapping could prove a useful complement to anatomic imaging. It has been found that the use of a mean ADC value greater than $\left(2.5 \times 10^{-3} \mathrm{~mm}^{2} / \mathrm{s}\right)$ yielded a sensitivity of $80 \%$ and a specificity of $100 \%$ in the diagnosis of benign cystic lesions, indicating that no soft tissue neoplasms are missed with DWI and ADC mapping [19].

Many previous studies reported that not all benign soft tissue tumors have a large extracellular space and not all malignant soft tissue tumors are more cellular than benign tumors [17]. In our study, there was an overlap in the recorded mean ADC values between some benign and malignant masses. The pediatric patients with benign masses demonstrating restricted diffusion 
Table 2 Comparison of DW-ADC values between benign and malignant masses

\begin{tabular}{|c|c|c|c|c|c|c|}
\hline \multirow[t]{2}{*}{ Pathology } & \multicolumn{5}{|c|}{$\mathrm{ADC} \times 10^{-3} \mathrm{~mm}^{2} / \mathrm{s}$} & \multirow[t]{2}{*}{ Test of significance } \\
\hline & $N$ & Mean & SD & Minimum & Maximum & \\
\hline Benign & 41 & 1.495 & 0.55 & 0.6 & 2.72 & \multirow{2}{*}{$\begin{array}{l}t=3.73 \\
p=0.001^{*}\end{array}$} \\
\hline Malignant & 21 & 0.449 & 0.27 & 0.20 & 0.74 & \\
\hline
\end{tabular}
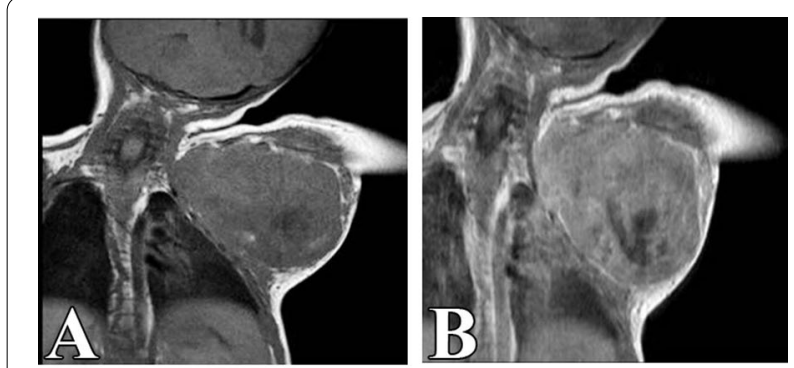

Coronal T1WI

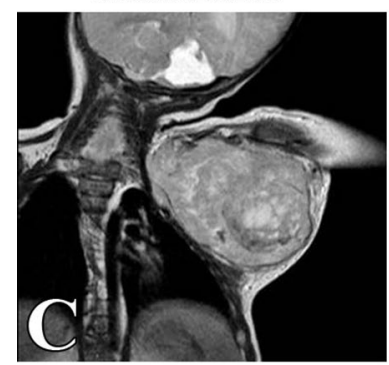

Coronal T2WI

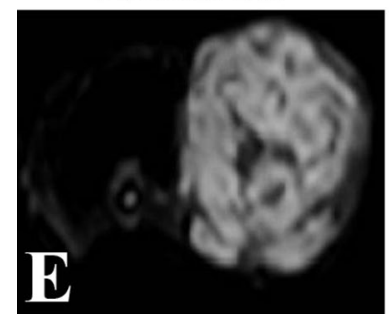

DWI
Coronal T1WI with contrast

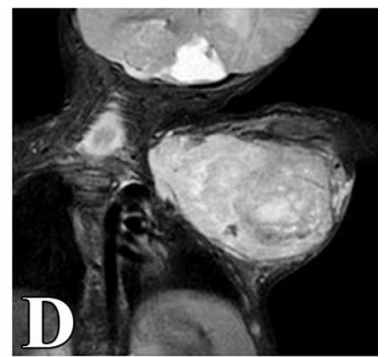

Coronal STIR

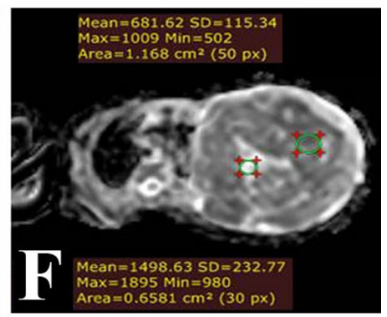

ADC MAP

Fig. 4 A 3-month-old female patient, presented with a large mass at the left axilla. A, B Coronal T1WI and coronal T1WI with contrast:

A large well-defined lobulated soft tissue mass of low signal intensity is seen involving predominantly the left axillary region and compressing chest wall posteromedially with no intervening fat planes between the mass and left lung apex. It is seen lateral abutting head of humerus with areas of cystic necrosis appear as small foci of low signal intensity. Heterogeneous enhancement noted in post-contrast series. C, D Coronal T2WI and coronal STIR: The mass shows heterogeneous high signal with small foci of high signal intensity representing areas of break down. E Axial DWI: The mass elicits high signal intensity. $\mathbf{F}$ Axial ADC map: The mass elicits low signal intensity with a mean ADC value of solid portion $\left(0.681 \times 10^{-1}\right.$ ${ }^{3} \mathrm{~mm}^{2} / \mathrm{s}$ ). Pathological result was a case of rhabdomyosarcoma

like malignancy were patients with abscess (mean ADC value $=0.6810 \pm 0.11314 \times 10^{-3} \mathrm{~mm}^{2} / \mathrm{s}$ ) and patients with PVNS (mean ADC value $=1.0580 \times 10^{-3} \mathrm{~mm}^{2} / \mathrm{s}$ ).

Our results matched with the previous study where the mean ADC value for soft tissue abscess was $0.877 \times 10^{-3} \mathrm{~mm}^{2} / \mathrm{s}$. This could be explained by the high viscosity pus within abscesses that contains inflammatory cells, cellular debris, bacteria and proteins slowing water diffusion [20].

In addition, several theories are plausible for low ADC values in PVNS. The presence of hemosiderin and other blood products in these masses likely affects the ADC measurements. Even if great care was taken to avoid sampling of clearly hemorrhagic components, it is possible that microscopic areas of hemorrhagic products were present also in the sampled pixels and affected ADC values [21]. Other potential reasons for low ADC measurements in PVNS include the nature of intralesional matrix or presence of hypercellular components, nodulated and villose proliferation of the synovium with thickened synovial rinds of hemosiderin-laden tissue [9, 22].

A large number of studies have yielded conflicting results and have found different cutoff ADC values between benign and malignant masses although the most discriminating ADC value appears to be near $\left(1 \times 10^{-3} \mathrm{~mm}^{2} / \mathrm{s}\right)$. The reason of this conflicting result in previous studies can be explained by the variations in histopathological types of neoplasms, differences in DWI techniques used (equipment, $b$ values and DWI sequences). However, all previous studies confirmed the fact that aggressive tumor usually records a very low ADC [23].

Regarding the ROC analysis in this study, the cutoff point for the mean $\mathrm{ADC}$ value was $0.88 \times 10-3 \mathrm{~mm}^{2} / \mathrm{s}$ with sensitivity of $100.0 \%$, specificity of $92.3 \%$ and diagnostic accuracy of $93.3 \%$. This recorded cutoff point was lower than that of previous study [9] in which the cutoff value was $1.235 \times 10^{-3} \mathrm{~mm}^{2} / \mathrm{s}$; however, our study recorded higher sensitivity, specificity and accuracy in comparison with their results that were $73 \%$ sensitivity, $91.7 \%$ specificity and $80.3 \%$ overall accuracy. Other study reported that malignant tumors tend to exhibit a lower mean ADC value than that of benign soft tissue tumors and proposed using a threshold mean ADC value of $1.34 \times 10^{-3} \mathrm{~mm}^{2} / \mathrm{s}$ [10]. The ROC analysis in another study [8] yielded nearly the same area under curve AUC that was $0.89 \times 10^{-3} \mathrm{~mm}^{2} / \mathrm{s}$ and a fairly close cutoff value for mean ADC which was 
Table 3 DW-ADC values distribution according to pathology results among the studied cases

\begin{tabular}{|c|c|c|c|c|c|}
\hline \multirow[t]{2}{*}{ Pathology } & \multicolumn{5}{|c|}{ DW-ADC $\times 10^{-3} \mathrm{~mm}^{2} / \mathrm{s}$} \\
\hline & $N$ & Mean & SD & Minimum & Maximum \\
\hline Lipoma & 9 & 1.1196 & .05497 & 1.04 & 1.21 \\
\hline Venous malformation(VM) & 2 & 1.9140 & .12162 & 1.83 & 2.00 \\
\hline Abscess & 6 & .6810 & .11314 & .60 & .76 \\
\hline Rhabdomyosarcoma (RMS) & 8 & .4498 & .27050 & .20 & .74 \\
\hline Pigmented villonodular synovitis (PVNS) & 2 & 1.0580 & 0.0 & 1.06 & 1.06 \\
\hline Lymphangioma & 5 & 2.2100 & .03960 & 2.18 & 2.24 \\
\hline Lipoblastoma/atypical lipoma & 1 & 1.00 & 0.0 & 1.00 & 1.00 \\
\hline Hemangioma & 8 & 1.6538 & .06664 & 1.56 & 1.72 \\
\hline Fibroma & 4 & 1.7895 & .07849 & 1.73 & 1.84 \\
\hline Baker's cyst & 4 & 2.6440 & .10889 & 2.57 & 2.72 \\
\hline Ewing sarcoma & 7 & 0.5978 & .36040 & .30 & .86 \\
\hline Lymphoma & 6 & .4937 & .34210 & .20 & .82 \\
\hline Total & 62 & 1.3560 & .62812 & .20 & 2.72 \\
\hline Test of significance & \multicolumn{5}{|c|}{$\begin{array}{l}F=80.16 \\
P<0.001^{*}\end{array}$} \\
\hline
\end{tabular}

Statistically significant (if $P<0.001 *$ )

Table 4 The validity of DW-ADC in differentiating benign from malignant masses

\begin{tabular}{lllllllll}
\hline & AUC $(\mathbf{9 5 \%} \mathrm{Cl})$ & $\boldsymbol{P}$ & Cut off point & Sensitivity (\%) & Specificity (\%) & PPV (\%) & NPV (\%) & Accuracy (\%) \\
\hline Mean ADC $\left(\times 10^{-3} \mathrm{~mm}^{2} / \mathrm{s}\right)$ & $0.981(0.935-1.03)$ & $\leq 0.001^{*}$ & 0.88 & 100.0 & 9.23 & 66.7 & 100.0 & 93.3 \\
\hline
\end{tabular}

$A U C$ area under curve, $P P V$ positive predictive value, $N P V$ negative predictive value, $\mathrm{Cl}$ confidence interval

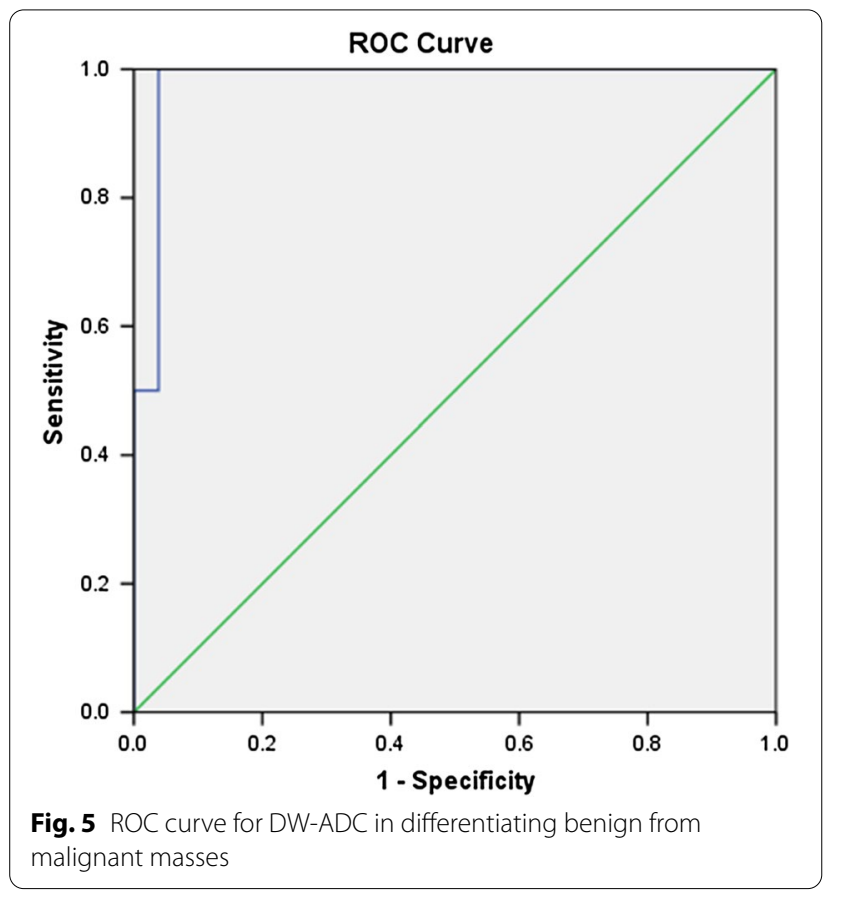

$\leq 1.03 \times 10^{-3} \mathrm{~mm}^{2} / \mathrm{s}$, but with relatively lower sensitivity $(90 \%)$ and specificity (91\%) than those of our study.

The limitations of this study were that the number of pediatric patients especially those with malignant tumors was limited in this study. We did not perform a histogram analysis or calculate perfusion effects. At last, we did not obtain inter-observer variations of measurement of ADC values.

\section{Conclusion}

In pediatric patients, DWI (with quantitative ADC mapping) is an innovative valuable noninvasive imaging technique for characterization of musculoskeletal soft tissue masses and discrimination between benign and malignant masses. It is particularly suitable for the inconclusive cases of pediatric population without urgent need for invasive diagnostic procedures. 


\begin{abstract}
Abbreviations
MRI: Magnetic resonance imaging; DWI: Diffusion-weighted imaging; ADC: Apparent diffusion coefficient; SD: Standard deviation; T1WI:T1-weighted imaging; T2WI:T2-weighted imaging; STIR: Short inversion recovery; EPI: Echo planner imaging; TR: Repetition time; TE: Echo time; FOV: Field of view; ROI: Region of interest; FNAC: Fine needle aspiration cytology; ROC: Receiver operating characteristic; PPV: Positive predictive value; NPV: Negative predictive value; AUC: Area under curve; Cl: Confidence interval; PVNS: Pigmented villonodular synovitis.
\end{abstract}

\section{Acknowledgements}

Not applicable.

\section{Authors' contributions}

ME revised the collected data and the manuscript. SO and DM analyzed the MRI images of all patients. ME and DM wrote the manuscript and performed the statistical analysis. All authors read and approved the final manuscript.

\section{Funding}

No funding resources.

\section{Availability of data and material}

The datasets used and/or analyzed during the current study are available from the corresponding author on reasonable request.

\section{Declarations}

\section{Ethics approval and consent to participate}

The study was approved by our institution's ethics committee (Mansoura Faculty of Medicine Institutional Research Board) (ethics committee reference number is MS/17.07.61), and all patients gave their written informed consent before inclusion in the study.

\section{Consent for publication}

Written informed consent for the publication of these data was obtained from the parents of the patients or from their legal guardian.

\section{Competing interests}

The authors declare that they have no competing interests.

\section{Author details}

${ }^{1}$ Faculty of Medicine, Mansoura University, Mansoura, Egypt. ${ }^{2}$ Resident of Diagnostic Radiology, Ministry of Health, Mansoura, Egypt.

Received: 30 July 2021 Accepted: 16 October 2021

Published online: 29 October 2021

\section{References}

1. De Schepper AM, De Beuckeleer L, Vandevenne J, Somville J (2000) Magnetic resonance imaging of soft tissue tumors. Eur Radiol 10(2):213-223

2. Tranvinh $\mathrm{E}$, Yeom KW, Iv M (2015) Imaging neck masses in the neonate and young infant. In: Seminars in ultrasound, CT and MRI, vol 36, no 2, pp 120-137

3. Piccolo CL, Galluzzo M, lanniello S, Trinci M, Russo A, Rossi E, Miele V (2017) Pediatric musculoskeletal injuries: role of ultrasound and magnetic resonance imaging. Musculoskelet Surg 101(1):85-102

4. Wang T, Wu X, Cui Y, Chu C, Ren G, Li W (2014) Role of apparent diffusion coefficients with diffusion-weighted magnetic resonance imaging in differentiating between benign and malignant bone tumors. World J Surg Oncol 12(1):365

5. Choi YJ, Lee IN, Song YS, Kim Jl, Choi K, Song JW (2019) Diagnostic performance of diffusion-weighted (DWI) and dynamic contrast-enhanced (DCE) MRI for the differentiation of benign from malignant soft-tissue tumors. J Magn Reson Imaging 50:798-809
6. Subhawong TK, Jacobs MA, Fayad LM (2014) Insights into quantitative diffusion-weighted MRI for musculoskeletal tumor imaging. Am J Roentgenol 203(3):560-572

7. Ahlawat S, Fritz J, Morris CD, Fayad LM (2019) Magnetic resonance imaging biomarkers in musculoskeletal soft tissue tumors: review of conventional features and focus on nonmorphologic imaging. J Magn Reson Imaging 50:11-27

8. Neubauer H, Evangelista L, Hassold N, Winkler B, Schlegel PG, Köstler H, Beer M (2012) Diffusion-weighted MRI for detection and differentiation of musculoskeletal tumorous and tumor-like lesions in pediatric patients. World J Pediatr 8(4):342-349

9. Hassanien OA, Younes RL, Dawoud RM (2018) Diffusion weighted MRI of soft tissue masses: can measurement of ADC value help in the differentiation between benign and malignant lesions? Egypt J Radiol Nucl Med 49(3):681-688

10. Razek A, Nada N, Ghaniem M, Elkhamary S (2012) Assessment of soft tissue tumours of the extremities with diffusion echoplanar MR imaging. Radiol Med (Torino) 117(1):96-101

11. Ahlawat S, Fayad LM (2015) De novo assessment of pediatric musculoskeletal soft tissue tumors: beyond anatomic imaging. Pediatrics 136(1):e194-e202

12. Subhawong TK, Wang X, Durand DJ, Jacobs MA, Carrino JA, Machado AJ, Fayad LM (2012) Proton MR spectroscopy in metabolic assessment of musculoskeletal lesions. Am J Roentgenol 198(1):162-172

13. Pekcevik Y, Kahya MO, Kaya A (2015) Characterization of soft tissue tumors by diffusion-weighted imaging. Iran J Radiol 12(3):e15478

14. Lee SK, Jee WH, Jung CK, Chung YG (2020) Multiparametric quantitative analysis of tumor perfusion and diffusion with 3T MRI: differentiation between benign and malignant soft tissue tumors. BJR 93(1115):20191035

15. Zou Y, Wang OD, Zong M, Zou YF, Shi HB (2016) Apparent diffusion coefficient measurements with diffusion-weighted imaging for differential diagnosis of soft-tissue tumor. J Cancer Res Ther 12(2):864-872

16. Nassif MA, Abdul Rahim SA, Hussien RZ (2017) The role of diffusionweighted MRI in the characterization of musculoskeletal soft tissue tumors. Egypt J Hosp Med 69(2):1895-1900

17. Khedr SA, Hassaan MA, Abdelrazek NM, yehia Sakr, A. (2012) Diagnostic impact of echo planar diffusion-weighted magnetic resonance imaging (DWI) in musculoskeletal neoplastic masses using apparent diffusion coefficient (ADC) mapping as a quantitative assessment tool. Egypt J Radiol Nucl Med 43(2):219-226

18. Romeih M, Raafat T, Khalaf M (2018) The diagnostic value of diffusionweighted magnetic resonance imaging in characterization of musculoskeletal soft tissue tumors. Egypt J Radiol Nucl Med 49(2):400-407

19. Subhawong TK, Jacobs MA, Fayad LM (2014) Diffusion-weighted MR imaging for characterizing musculoskeletal lesions. Radiographics 34(5):1163-1177

20. Chun CW, Jung JY, Baik JS, Jee WH, Kim SK, Shin SH (2018) Detection of soft-tissue abscess: comparison of diffusion-weighted imaging to contrast-enhanced MRI. J Magn Reson Imaging 47:60-68

21. Ashikyan O, Chalian M, Moore D, Xi Y, Pezeshk P, Chhabra A (2019) Evaluation of giant cell tumors by diffusion weighted imaging-fractional ADC analysis. Skelet Radiol 48(11):1765-1773

22. Li Y, Lou H, Wang R, Wang C, Zhu W, Xia L, Zhu D (2003) MRI-pathologic correlation of pigmented villonodular synovitis. Chin J Radiol 37(6):493-498

23. Dallaudière $B$, Lecouvet $F$, Berg BV, Omoumi P, Perlepe V, Cerny M, Larbi A (2015) Diffusion-weighted MR imaging in musculoskeletal diseases: current concepts. Diagn Interv Imaging 96(4):327-340

\section{Publisher's Note}

Springer Nature remains neutral with regard to jurisdictional claims in published maps and institutional affiliations. 